\title{
DIE DEKLARATION VON MONTEVIDEO \\ ÜBER DAS SEERECHT VOM 8. JULI 1970 \\ IM LICHTE DER BEMÜHUNGEN \\ UM EINE DRITTE SEERECHTSKONFERENZ
}

\author{
Von Max Ivers Kehden
}

In der Zeit vom 4. bis zum 8. Juli 1970 fand auf Einladung der Regierung Uruguays in Montevideo eine Konferenz über Fragen des internationalen öffentlichen Seerechts statt, die dem erklärten Ziel diente, unter den Küstenstaaten, welche Hoheitsrechte über Meereszonen bis zu 200 sm Breite beanspruchen, Grundsätze gemeinschaftlichen Handelns zur Verteidigung dieser Rechte ${ }^{1}$ abzustimmen. Teilnehmer waren die neun Staaten Mittel- und Südamerikas, welche ausschließliche Hoheitsrechte über $200 \mathrm{sm}$ breite Meereszonen in Anspruch nehmen, nämlich:

1. Argentinien: Küstenmeer ${ }^{2}$,

2. Brasilien, Küstenmeer ${ }^{3}$,

3. Chile: Fischereizone ${ }^{4}$,

4. Ecuador: Küstenmeer ${ }^{5}$,

5. El Salvador: Küstenmeer ${ }^{6}$,

6. Nicaragua: Fischereizone ${ }^{7}$,

7. Panama: Küstenmeer ${ }^{8}$,

8. Peru: Küstenmeer ${ }^{9}$,

9. Uruguay: Küstenmeer ${ }^{10}$.

Costa Rica ${ }^{11}$ und Honduras ${ }^{12}$ beanspruchen lediglich Schutz- und Überwachungsrechte in einer Zone von $200 \mathrm{sm}$ Breite zur Erhaltung der dortigen Meeresschätze. Als Beobachter nahmen an der Konferenz die in Montevideo akkreditierten Missionschefs von Bolivien und Kolumbien teil.

Hauptergebnis der Konferenz war die Annahme einer Deklaration über Prinzipien des Seerechts ${ }^{13}$, die angeblich die wichtigsten Rechte der Küstenstaaten hinsichtlich der Verfügung über die Naturschätze der an ihr Staatsgebiet angrenzenden Meereszonen zusammenfaßt. Nun bilden jedoch Inhalt und Umfang der küsten-

$1 \mathrm{Da}$ die Rechtswidrigkeit der Inanspruchnahme derart breiter Meereszonen außer Zweifel steht, wäre es richtiger gewesen, wenn man in der amtlichen Verlautbarung des uruguayischen Außenministeriums über die Konferenz vom 8. 7. 1970 (abgedruckt in: El Boletin del Ministerio de Relaciones Exteriores vom 10. 7. 1970, S. 7) von der Durchsetzung dieser Ansprüche gesprochen hätte.

2 Gesetz Nr. 17.094 vom 29. 12. 1966, Art. 1; spanischer Text: La Nación vom 9. 1. 1967.

3 Gesetzesdekret Nr. 1098 vom 25. 3. 1970, Art. 1; amtlicher Text: Diário Oficial vom 30. 3. 1970.

4 Gemeinsame Seezonen-Erklärung der Staaten Chile, Ecuador und Peru vom 18. 8. 1952; englischer Text: Laws and Regulations on the Regime of the Territorial Sea, United Nations Legislative Series, Bd. 6, New York 1957 (im folgenden zitiert als ST/LEG/SER. B/6), S. 723.

5 Código Civil, Art. 633 i. d. F. des Dekrets Nr. 1542 vom 10. 11. 1966; amtlicher Text: Registro Oficial Nr. 158 vom 11. 11. 1966.

6 Verfassung vom 8. 1. 1962, Art. 8; spanischer Text: Constitución Política de la República de El Salvador 1962, San Salvador 1962, S. 4.

7 Dekret Nr. 1-L vom 5. 4. 1965, Art. 1; amtlicher Text: La Gaceta Nr. 82 vom 8. 4. 1965.

8 Gesetz Nr. 31 vom 2. 2. 1967, Art. 1; amtlicher Text: Gaceta Oficial Nr. 15.803 vom 14. 2.1967.

9 Dekretgesetz Nr. 17752 vom 24. 7. 1969, Art. 1, 4a; amtlicher Text: El Peruano vom 25. 7. 1969 , S. 6.

10 Gesetz Nr. 13.833 vom 29. 12. 1969, Art. 2; amtlicher Text: Diario Oficial vom 5. 1. 1970.

11 Gesetzesdekret Nr. 803 vom 2. 11. 1949, Art. 4; englischer Text: Laws and Regulations on the Regime of the High Seas, United Nations Legislative Series, Bd. 1, New York 1951 (im folgenden zitiert als ST/LEG/SER. B/1), S. 9

12 Dekret Nr. 25 vom 17. 1. 1951, Art. 2, 3; englischer Text: ST/LEG/SER. B/6, S. 22.

13 Amtlicher Text: uruguayisches Boletin del Ministerio de Relaciones Exteriores vom 10. 7. 1970, S. 9; deutsche Ubersetzung des Autors abgedruckt auf S. 531. 
staatlichen Rechte zur Zeit den am heftigsten umstrittenen Fragenkomplex des Seevölkerrechts überhaupt. Die Vermutung liegt daher nahe, daß die Verfasser der Deklaration etwas anderes wollten als lediglich - wie es nach deren Wortlaut den Anschein hat - geltendes Völkerrecht feststellen. Andererseits hat es sich in der Nachkriegszeit wiederholt gezeigt, daß die Staaten Lateinamerikas unter den Entwicklungsländern eine Führungsrolle bei der Herausbildung und Formulierung von Standpunkten zu strittigen Fragen des Seevölkerrechts einnehmen ${ }^{14}$. Sie sind also der wichtigste Gesprächspartner der Industriestaaten bei der Lösung der anstehenden seerechtlichen Probleme. Es erscheint daher im Hinblick auf die Bemühungen um eine dritte Seerechtskonferenz nützlich zu klären, was es mit der Deklaration in Wirklichkeit auf sich hat.

\section{I.}

Das wird schon etwas deutlicher, wenn man sich vergegenwärtigt, welche Themen auf der Konferenz außerdem behandelt wurden. Von den Beratungsgegenständen sind in diesem Zusammenhang zu nennen:

1. Abstimmung der Grundlagen der Antworten der auf der Konferenz vertretenen Staaten auf eine Anfrage des Generalsekretärs der Vereinten Nationen hinsichtlich der Einberufung einer internationalen Konferenz über Hauptfragen des Seevölkerrechts ${ }^{15}$,

2. Das gleiche im Hinblick auf Anfragen der Sowjetunion und der Vereinigten Staaten betreffend die Abhaltung einer internationalen Konferenz über die Festlegung der äußersten Breite des Küstenmeeres, des freien Durchfahrtsrechts durch Meerengen und der Regelung des Fischfangs außerhalb des Küstenmeeres und der Anschlußzone ${ }^{16}$,

3. Erörterung der Fragen betreffend die Rechtsordnung des Meeresbodens außerhalb der Grenzen nationaler Hoheit ${ }^{17}$,

14 Besonders deutlich wird diese Führungsrolle in letzter Zeit bei der Erörterung der Frage der Nutzung des Tiefseebodens in den Vereinten Nationen. Dort ist es den Staaten Lateinamerikas in der 24. Sitzungsperiode der Vollversammlung gelungen, unter der Devise der Solidarität der Entwicklungsländer eine Konfrontation dieser Staaten im Verhältnis zu den Industrienationen herbeizuführen und die Annahme einer Resolution durchzusetzen, die ein Moratorium hinsichtlich der Ausbeutung der Naturschätze des Tiefseebodens, aber nicht hinsichtlich der Ausdehnung der Ansprüche der Küstenstaaten in den an ihr Staatsgebiet angrenzenden Meereszonen beinhaltet; vgl. Resolution 2574 D (XXIV) vom 15.12 . 1969 , amtlicher Text: U. N. Doc. A/RES/2574 (XXIV) vom 15. 1. 1970, S. 5. Kurz darauf dehnten Uruquay (am 29. 12. 1969, vgl. Anm. 10) und Brasilien (am 25. 3. 1970, vgl. Anm. 3) ihr Küstenmeer auf 200 sm aus, eine Verhaltensweise, die im Verhältnis zu vielen Entwicklungsländern kaum als solidarisch anzusehen ist.

15 Außer der in Anm. 14 genannten Resolution nahm die 24. UN-Vollversammlung am 15.12 .1969 die Resolution 2574 A (XXIV) an, in welcher der Generalsekretär aufgefordert wird, die Auffassungen der Mitgliedstaaten hinsichtlich der baldigen Einberufung einer Seerechtskonferenz über die Fragen der Rechtsordnung des Hohen Meeres, des Festlandsockels, des Küstenmeeres und der Anschlußzone, der Fischerei und Erhaltung der lebenden Schätze des Hohen Meeres, mit dem besonderen Ziel der Vereinbarung einer präzisen und allgemein anerkannten. Abgrenzung des Meeresbodens und -untergrundes außerhalb der Grenzen nationaler Hoheit zu ermitteln; vgl. U. N. Doc. A/RES/2574 (XXIV) vom 15. 1. 1970 , S. 1.

16 Die Sowjetunion und die Vereinigten Staaten arbeiten seit einiger Zeit auf eine 3 . Seerechtskonferenz hin, in der die äußerste Breite des Küstenmeeres auf $12 \mathrm{sm}$ festgelegt, das Recht der freien Durchfahrt durch Meerengen garantiert und Vorzugsrechte der Küstenstaaten hinsichtlich der Fischerei in den an ihr Küstenmeer angrenzenden Meereszonen geschaffen werden sollen; vgl. das Statement des amerikanischen Außenministeriums vom 25. 2. 1970 (amtlicher Text: The Department of State Bulletin $\mathrm{Nr}$. 1603 vom 16. 3. 1970, S. 343).

17 Das Problem der Nutzung des außerhalb der Grenzen nationaler Hoheitsgewalt liegenden Tiefseebodens beschäftigt seit Mitte der sechziger Jahre in immer stärkerem Maße nationale und internationale Gremien und war in der Zeit vom 28. 6. bis 3. 7. 1970 Gegenstand eines großangelegten internationalen Kongresses in Malta; vgl. den Tagungsbericht von Kehden - Steinicke in: Hansa, Bd. 107 (1970), S. 1612-1616. Es ist als der Hauptantrieb für die gegenwärtigen Bestrebungen zur Neuordnung des Seevölkerrechts anzusehen. Seine Lösung wird jedoch dadurch zusätzlich erschwert, daß es in engem Zusammenhang mit den Problemen der Entwicklungshilfe steht. 
4. Beratung des Problems der Verhinderung der Lagerung von Atom- und Massenvernichtungswaffen auf dem Meeresboden ${ }^{18}$,

5. Neubestimmung des Festlandsockelbegriffs' ${ }^{19}$.

Es wurde also fast das ganze Spektrum aktueller Fragen des internationalen öffentlichen Seerechts erörtert. In diesem größeren Zusammenhang ist auch die Deklaration von Montevideo zu sehen, und zwar als ein massiver Versuch, den in diesem Bereich gegenwärtig stattfindenden Willensbildungsprozeß im Sinne der Interessen der Signatarstaaten zu beeinflussen.

\section{II.}

Eine kurze Analyse der sechs in der Deklaration aufgezählten Grundprinzipien des Seerechts soll diese Qualifikation erhärten und gleichzeitig - soweit möglich — Aufschluß über die Ziele der Signatarstaaten geben:

1. Das in Art. 1 behauptete Recht der Küstenstaaten zur Verfügung über die Naturschätze des an ihre Küsten angrenzenden Meeres, Meeresbodens und -untergrundes ist in dieser Unbestimmtheit nicht Bestandteil des geltenden Seevölkerrechts. Letzteres gibt den Küstenstaaten ausschließliche Nutzungsrechte lediglich im Bereich ihres Küstenmeeres, wozu auch dessen Boden und Untergrund zu rechnen ist ${ }^{20}$, und ihres Festlandsockels, dem das darüberliegende epikontinentale Meer nicht zuzurechnen ist ${ }^{21}$. Die Naturschätze des Hohen Meeres einschließlich seines Bodens und Untergrundes, soweit sie nicht zum Festlandsockel eines Küstenstaates gehören, stehen allen Staaten zur Nutzung offen und unterliegen deshalb nicht der Verfügungsgewalt der Küstenstaaten ${ }^{22}$. Art. 1 der Deklaration gibt also nicht geltendes Völkerrecht wieder. Er dient vielmehr in Wirklichkeit dazu, die uferstaatlichen Rechre auf das Hohe Meer auszudehnen, und zwar über die Festlandsockelrechte hinaus. Letzteres folgt nicht nur aus der besonderen Erwähnung der Festlandsockelrechte in Art. 4 der Deklaration, sondern vor allem auch aus deren Art. 2, der wichtigsten und gefährlichsten Bestimmung der Deklaration.

18 Bekanntlich war auch der revidierte amerikanisch-sowjetische Vertragsentwurf über das Verbot der Lagerung von Atom- und Massenvernichtungswaffen auf dem Meeresboden vom 23. 4. 1970 in der in Genf tagenden Konferenz des UN-Abrüstungsausschusses auf Ablehnung gestoßen, und zwar nicht zuletzt deshalb, weil einige lateinamerikanische Ausschußmitglieder fürchteten, die in dem Vertragsentwurf vorgesehene 12-sm-Zone präjudiziere ihre Ansprüche auf Hoheitsrechte in der 200-sm-Zone. Inzwischen haben die Sowjetunion und die Vereinigten Staaten am 1. 9. 1970 einen nochmals revidierten Vertragsentwurf vorgelegt, der praktisch von jeder Partei in einem anderen, ihren Interessen entsprechenden Sinne interpretiert werden kann (deutscher Text: Archiv der Gegenwart 1970, S. 15699). Das dürfte auch ein Grund sein, weshalb diesem Entwurf sogar Argentinien und Brasilien zugestimmt haben, so daß er inzwischen der UN-Vollversammlung zur weiteren Beratung zugeleitet werden konnte; vgl. Die Welt vom 4. 9. 1970.

19 Auch das Problem der Abgrenzung des Festlandsockels, welches in Art. 1 der Genfer Konvention über den Festlandsockel vom 29. 4. 1958 (im folgenden: FSK) völlig unzulänglich gelöst worden ist, hat besondere Aktualität durch die bevorstehende Nutzung des Tiefseebodens erhalten; denn die Grenze des Tiefseebodens fällt in der Regel mit der des Festlandsockels zusammen. Konkreter Anlaß für die diesbezüglichen Beratungen in Montevideo dürfte daher der Vorschlag Präsident Nixons vom 23. 5. 1970 (amtlicher Text: The Department of State Bulletin Nr. 1616 vom 15. 6. 1970, S. 737) gewesen sein. im Rahmen einer vertraglichen Regelung über die Nutzung des Tiefseebodens die Festlandsockelrechte der Küstenstaaten auf die 200-m-Tiefenlinie zu beschränken.

$20 \mathrm{Vgl}$. Art. 2 der Genfer Konvention über das Küstenmeer und die Anschlußzone vom 29. 4. 1958 (im folgenden: KMK), der lediglich Ausdruck eines entsprechenden Völkergewohnheitsrechts ist.

$21 \mathrm{Vgl}$. Art. 3 der FSK, der insofern lediglich die komplementäre Norm zu dem inzwischen gewohnheitsrechtlich geltenden Art. 1 der FSK bildet.

22 Vgl. Art. 2 der Genfer Konvention über das Hohe Meer vom 29. 4. 1958 (im folgenden: HMK), der lediglich bereits geltendes Völkergewohnheitsrecht wiedergibt. Ob der in Art. 2 normierte Grundsatz der Freiheit der Meere auch für den Boden und Untergrund des Hohen Meeres gilt, ist allerdings bestritten. Man wird diese Frage jedoch vorbehaltlich der Einschränkungen durch die Festlandsockelrechte der Küstenstaaten bejahen müssen; vgl. Kehden, M. I.: Die Vereinten Nationen und die Nutzung des Bodens und Untergrundes des Hohen Meeres außerhalb der Grenzen nationaler Hoheitsgewalt, in: Verfassung und Recht in Übersee, Bd. 2 (1969), S. 131-167 (160 ff.), mit weiteren Nachweisen. Die Frage, ob die Küstenstaaten inzwischen gewohnheitsrechtlich ausschließliche Fischereirechte in den an ihr Küstenmeer angrenzenden Teilen des Hohen Meeres beanspruchen können, kann in diesem Zusammenhang offenbleiben, da solche Rechte jedenfalls nicht über die 12-sm-Zone hinausgehen. 
2. Nach Art. 2 soll nämlich der Küstenstaat das Recht haben, die seewärtigen Grenzen seiner Souveränität einseitig festzusetzen, und dabei nur an derart unbestimmte Abgrenzungsmerkmale wie seine geographischen und geologischen Besonderheiten u. ä. gebunden sein, was darauf hinauslaufen würde, daß das Hohe Meer zur Disposition der Küstenstaaten steht. Eine derartige Regel stände jedoch in krassem Gegensatz zu Art. 2 der Genfer Konvention über das Hohe Meer vom 29. April 1958, wonach kein Staat das Recht für sich in Anspruch nehmen kann, Teile des Hohen Meeres seiner Souveränität zu unterstellen ${ }^{23}$. Die einseitige Festsetzung der seewärtigen Grenzen der Souveränität eines Küstenstaates durch diesen selbst verstößt also gegen das Völkerrecht, soweit dadurch wie bei den meisten Ansprüchen auf die 200-sm-Zone Teile des Hohen Meeres zum Bestandteil des Küstenmeeres des betreffenden Staates gemacht und damit dem Gemeingebrauch aller Staaten entzogen werden.

Wann das der Fall ist, ist allerdings höchst umstritten. Insbesondere hat man sich auf den Genfer Seerechtskonferenzen über die höchstzulässige Breite des Küstenmeeres nicht einigen können. Sie richtet sich daher nach den Grundsätzen des Völkergewohnheitsrechts. Aber auch diese führen im Augenblick nur mit Vorbehalt zu einer Antwort; denn wir befinden uns unabhängig von den übersteigerten Ansprüchen einiger lateinamerikanischer Staaten in einem Prozeß der Ausdehnung küstenstaatlicher Rechte, dessen endgültiges Ergebnis noch nicht abzusehen ist. In diesem Zusammenhang sei daher lediglich vermerkt, daß eine Tendenz zur Ausdehnung des Küstenmeeres auf $12 \mathrm{sm}$ Breite unverkennbar ist, weitergehende Ansprüche aber in absehbarer Zeit an dem Widerstand der Großmächte scheitern dürften ${ }^{24}$.

Nicht zuletzt deshalb wird eine Regel wie Art. 2 der Deklaration aller Voraussicht nach auch in Zukunft nicht Bestandteil des Seevölkerrechts werden. Würde sie doch mangels einer präzisen Begrenzung der Rechte der Küstenstaaten nicht nur Ansprüche auf ein Küstenmeer von mehr als $12 \mathrm{sm}$ Breite, sondern auch auf ein Küstenmeer, das sogar breiter als $200 \mathrm{sm}$ ist, rechtfertigen können, ja selbst die völlige Nationalisierung der Ozeane erlauben und dadurch den Gemeingebrauch aller Staaten an dem Hohen Meer, die Freiheit der Meere, in Frage stellen. Diese Konsequenzen dürften auch den Signatarstaaten bewußt sein. Es handelt sich also bei Art. 2 um eine in eine Rechtsregel gekleidete politische Forderung, von der vermutlich auch die in Montevideo vertretenen Staaten wissen, daß sie in dieser Form nicht durchsetzbar ist. Das legt die weitere Vermutung nahe, daß diese Staaten entweder die Taktik verfolgen, durch unannehmbare Forderungen die von anderen Staaten angestrebte dritte Seerechtskonferenz von vornherein scheitern zu lassen oder für diese Konferenz eine Verhandlungsposition aufzubauen, die es ihnen ermöglichen soll, ihre Hoheitsansprüche wenigstens zum größten Teil durchzusetzen.

23 Das ergibt sich eigentlich schon aus dem Begriff des Hohen Meeres selbst, denn als solches ist ja gerade der Teil der Weltmeere zu umschreiben, an den Souveränitätsrechte nicht begründet werden dürfen. Das Hohe Meer ist also nicht lediglich - wie es nach Art. 1 der HMK den Anschein haben könnte, wenn Art. 2 nicht wäre - der Rest, der nach Abzug der Küstenmeere und Binnengewässer von den Weltmeeren nachbleibt; vgl. Krüger, Herbert: Hohe See, in: Strupp-Schlochauer: Wörterbuch des Völkerrechts, 2 . Aufl., Bd. 1, Berlin 1960 (im folgenden zitiert als Wörterbuch I), S. 791-795 (791). Etwas anderes gilt lediglich für den Fall, daß ein Staat die höchstzulässige Küstenmeerbreite nicht in Anspruch nimmt. Diesem wird man das Recht nicht absprechen können, jederzeit einseitig sein Küstenmeer bis zur höchstzulässigen Breite auszudehnen und den betreffenden Teil des Meeres, der bis dahin Hohes Meer war, in sein Küstenmeer einzuverleiben.

24 Diese Tendenz wird in gewissem Umfang begünstigt durch Art. 24 der KMK, wonach sich die an das Küstenmeer angrenzende Anschlußzone nicht weiter als $12 \mathrm{sm}$ über die Ausgangslinie hinaus erstredken darf, von der aus die Breite des Küstenmeeres gemessen wird. Hieraus leiten einige Staaten nämlich das Redit her, auch das Küstenmeer selbst einseitig, und zwar sogar im Verhältnis zu Staaten, die nicht Vertragsparteien dieser Konvention sind, bis auf $12 \mathrm{sm}$ auszudehnen. 
3. Die zweite Alternative dürfte wahrscheinlicher sein. Sonst ist es nur schwer zu erklären, weshalb in Art. 3 ein Recht des Küstenstaates zur Erforschung und Erhaltung der lebenden Schätze des an sein Staatsgebiet angrenzenden Meeres und zum Erlaß von Vorschriften über den Fischfang in diesem Gebiet besonders herausgestellt wird. Ist es nämlich den Signatarstaaten ernst mit den in Art. 1 und 2 genannten "Rechten“, dann bedarf die in Art. 3 genannte Befugnis eigentlich keiner besonderen Erwähnung mehr. Wer die seewärtigen Grenzen seiner Souveränität einseitig festlegen und über die Naturschätze des an seine Küsten angrenzenden Meeres verfügen darf, kann selbstverständlich auch die lebenden Schätze dieser Meereszone erforschen und erhalten sowie ihren Fang durch Vorschriften regeln.

Anders verhält es sich, wenn ein Küstenstaat die in den ersten beiden Artikeln der Deklaration genannten "Rechte“ nicht, bzw. nicht in der behaupteten Form besitzt. Dann ergeben sich zwar die Rechte des Art. 3, soweit das Küstenmeer in Frage steht, aus der Gebietshoheit des Küstenstaates ${ }^{25}$, und soweit das Hohe Meer in Frage steht, hinsichtlich der Erforschung aus der Freiheit der Meere ${ }^{26}$. Ein Recht des Küstenstaates zur Regelung des Fischfangs auf dem an sein Küstenmeer angrenzenden Hohen Meer zwecks Erhaltung der lebenden Meeresschätze kennt das Völkerrecht jedoch nur im Rahmen der Genfer Konvention über die Fischerei und die Erhaltung der lebenden Schätze des Hohen Meeres von 29. April 1958, und auch dort nur unter bestimmten Voraussetzungen ${ }^{27}$. Hinter Art. 3 verbirgt sich also möglicherweise eine Minimalforderung für künftige Verhandlungen über eine Neugestaltung des Seevölkerrechts, nämlich auf Schaffung eines Rechts der Küstenstaaten, in den an ihr Küstenmeer angrenzenden Teilen des Hohen Meeres Hoheitsrechte zur Erhaltung der lebenden Meeresschätze auszuüben. Es läßt sich allerdings nur schwer vorstellen, daß die Signatarstaaten ihre Ansprüche auf ausschließliche Fischereirechte in den an ihr Küstenmeer angrenzenden Teilen des Hohen Meeres zugunsten einer derartigen Regelung fallen lassen werden.

4. Das in Art. 4 aufgeführte Recht der Küstenstaaten zur Erforschung, Erhaltung und Ausbeutung der Naturschätze ihrer Festlandsockel, soweit eine Ausbeutung möglich ist, entspricht - wenn man von der Weglassung der inzwischen durch die technische Entwicklung obsolet gewordenen 200-m-Tiefenlinie absieht — der Definition der Festlandsockelrechte in den Art. 1 und 2 der Genfer Konvention über den Festlandsockel vom 29. April 195828. Da diese Rechte heute unangefochtener Bestandteil des geltenden Seevölkerrechts sind, erhebt sich auch hier die Frage, weshalb sie in der Deklaration besonders genannt werden, zumal sie sich schon aus den Art. 1 und 2 der Deklaration ergeben. Wenn die Vermutung stimmt, daß der in Präsident Nixons Erklärung vom 23. Mai 1970 enthaltene Vorschlag zur Beschränkung der Festlandsockelrechte auf die 200-m-Tiefenlinie konkreter Anlaß für

$25 \mathrm{Da}$ das Küstenmeer heute dem Staatsgebiet zugerechnet wird, besitzt der Küstenstaat in diesem nahezu die gleichen Rechte wie auf seinem Landgebiet; vgl. Münch, Fritz: Küstengewässer, in: Strupp-Schlochauer: Wörterbuch des Völkerrechts, 2. Aufl., Bd. 2, Berlin 1961, S. 388-391 (390 f.).

26 Die Forschungsfreiheit ist zwar in Art. 2 der HMK nicht genannt. Diese Bestimmung enthält aber nur eine beispielhafte Aufzählung der Freiheiten.

$27 \mathrm{Vgl}$. Art. 7. Ob diese Bestimmung zwischenzeitlich allgemein verbindliches Völkerrecht geworden ist, kann in diesem Zusammenhang offenbleiben, da es den Signatarstaaten offenbar um weitergehende Befugnisse geht. Das gleiche gilt für Art. 13.

28 Eine Annäherung an diese Konvention deutet sich auch dadurch an, daß von einem Anspruch auf das epikontinentale Meer nicht mehr die Rede ist. 
die Beratungen in Montevideo über die Neubestimmung des Festlandsockelbegriffs war' ${ }^{29}$, dürfte der Grund gerade in der Weglassung der 200-m-Tiefenlinie zu suchen sein. Letztere ist dann nämlich als indirekte Absage an alle Bestrebungen zu verstehen, die vage Festlegung der äußeren Grenze des Festlandsockels in Art. 1 der FestlandsockelKonvention durch eine präzise Grenzziehung zu ersetzen, bzw. die Festlandsockelrechte entsprechend dem Vorschlag Präsident Nixons auf die 200-mTiefenlinie zu beschränken.

5. Auch das Recht zur Erforschung, Erhaltung und Ausbeutung der Naturschätze des Bodens und Untergrundes des Küstenmeeres, welches in Art. 5 der Deklaration angesprochen ist ${ }^{30}$, bedarf als anerkannter Bestandteil des internationalen öffentlichen Seerechts ${ }^{31}$ keiner neuerlichen Bekräftigung. Das gilt insbesondere dann, wenn wenigstens die Signatarstaaten selbst davon überzeugt sind, daß ein Küstenstaat so weitgehende Rechte wie die in Art. 1 und 2 der Deklaration behaupteten besitzt. Es ist deshalb nicht ausgeschlossen, daß die Signatarstaaten mit Art. 5 in Wirklichkeit andeuten wollen, sie würden bei zukünftigen Verhandlungen über die Begrenzung der küstenstaatlichen Rechte auf die ausschließliche Nutzung der Naturschätze des Meeresbodens und -untergrundes in der von ihnen beanspruchten 200-sm-Zone nicht verzichten, auch wenn man sich auf eine geringere Breite des Küstenmeeres einigen würde, bzw. sie würden letzterem nur zustimmen, wenn ihre Ansprüche auf die ausschließliche Nutzung der Bodenschätze in einer Zone dieser Breite anerkannt würden. Diese Deutung drängt sich jedenfalls auf, wenn man berücksichtigt, daß der eigentliche Zweck der Konferenz die Durchsetzung der Hoheitsansprüche über Meereszonen dieser Breite war und diese Ansprüche in erster Linie im Hinblick auf die Ausbeutung der Naturschätze dieser Meereszonen erhoben werden ${ }^{32}$.

6. Die Erwähnung des Rechts zum Erlaß von Vorschriften für die Schiffahrt und den Flugverkehr im Küstenmeer, bzw. im darüberliegenden Luftraum in Art. 6 schließlich erscheint an sich ebenfalls überflüssig, da es anerkannt ist, daß auch fremde Schiffe bei der Durchfahrt durch das Küstenmeer die Vorschriften des Küstenstaates beachten müssen ${ }^{33}$. Bemerkenswert ist aber die in Art. 6 enthaltene Einschränkung, daß diese Vorschriften die Freiheit der Schiffahrt und des Flugverkehrs nicht beeinträchtigen dürfen; denn nach geltendem Seevölkerrecht ist der Küstenstaat nur zur Duldung der friedlichen Durchfahrt verpflichtet ${ }^{34}$. Wenn die Signatarstaaten, die sich sonst in ihren Ansprüchen auf küstenstaatliche Rechte nicht gerade durch Bescheidenheit auszeichnen, dennoch in diesem Punkt mehr gewähren, als sie von ihrem eigenen Rechtsstandpunkt aus gewähren müßten, so deutet das darauf hin, daß auch ihnen die Maßlosigkeit des Anspruches auf ein Küstenmeer von $200 \mathrm{sm}$ Breite bewußt ist.

Der Umstand, daß nach Art. 6 der Deklaration die Freiheiten der Schiffahrt und des Flugverkehrs unberührt bleiben sollen, ist im übrigen auch aus folgendem

\footnotetext{
29 Vgl. Anm. 19.

30 Wörtlich wird allerdings nur von der Grenze gesprochen, bis zu der ein Küstenstaat seine njurisdicción ${ }^{\star}$ über das Meer ausübt.

31 Boden und Untergrund des Küstenmeeres unterliegen wie dieses selbst der Souveränität und damit der ausschließlichen Nutzung durch den Küstenstaat; vgl. Art. 2 der KMK.

$32 \mathrm{Vgl}$. The New York Times vom 26. 3. 1970 zur Ausdehnung des brasilianischen Küstenmeeres.

33 Vgl. Art. 17 der KMK.

$34 \mathrm{Vgl}$. Art. $14 \mathrm{ff}$. der KMK.
} 
Grunde interessant: Während die ersten Maßnahmen zur Ausdehnung des Küstenmeeres auf $200 \mathrm{sm}$ Breite diese Freiheiten überwiegend noch ausdrücklich unangetastet ließen ${ }^{35}$, differenziert das uruguayische Gesetz Nr. 13.833 (Ley de Pesca) vom 29. Dezember 1969 zwischen einer $12 \mathrm{sm}$ breiten inneren Zone und dem verbleibenden äuferen Teil des Küstenmeeres. In der inneren Zone wird lediglich noch das Recht der friedlichen Durchfahrt gewährt. Dagegen bleibt in der äußeren Zone die Freiheit der Schiffahrt unberührt ${ }^{36}$. Das brasilianische Gesetzesdekret Nr. 1098 vom 25. März 1970 hat auch diese Linie verlassen und gewährt in der ganzen 200-sm-Zone nur noch das Recht der friedlichen Durchfahrt ${ }^{37}$. Wenn nun aber in Art. 6 der Deklaration davon die Rede ist, daß die Freiheit der Schiffahrt im Küstenmeer unberührt bleiben müsse, so deutet das darauf hin, daß ein inhaltlich dem Küstenmeer im herkömmlichen Sinne völlig gleichzusetzender Souveränitätsanspruch wie der brasilianische nicht einmal die Unterstützung der in Montevideo vertretenen Staaten gefunden hat. Insofern könnte die Deklaration, obwohl sie verbal in Art. 2 noch über die Ansprüche der Signatarstaaten hinausgeht, in Wirklichkeit einen Wendepunkt in der Haltung der betreffenden Staaten andeuten und möglicherweise sogar ihre Bereitschaft signalisieren, bei zukünftigen Verhandlungen unter bestimmten Voraussetzungen die Küstenmeerbreite entsprechend den Vorstellungen der Großmächte auf $12 \mathrm{sm}$ zu beschränken.

\section{III.}

Damit ist die abschließende Frage angesprochen, welche Bedeutung für die weitere Entwicklung der Deklaration vermutlich zukommen wird. Zu deren Beantwortung genügt es allerdings nicht, die Deklaration in Beziehung zu den gegenwärtigen Diskussionen über die Reform des Seevölkerrechts zu setzen und von dort her zu interpretieren. Es ist vielmehr erforderlich, auch auf die geschichtlichen Wurzeln der Deklaration einzugehen. Da diese der Durchsetzung der Ansprüche der Signatarstaaten auf Hoheitsrechte in der 200-sm-Zone dient, sind die Ursprünge der Deklaration in den Ursachen für diese Ansprüche zu suchen.

Als deren auslösendes Moment wird übereinstimmend die Proklamation Nr. 2667 Präsident Trumans vom 28. September $1945^{38}$ angesehen, in welcher die Vereinigten Staaten erstmalig ausschließliche Nutzungsrechte an den Naturschätzen des ihren Küsten vorgelagerten Festlandsockels in Anspruch genommen haben. Das Gewicht der Maßnahme lag zunächst vor allem im binnenwirtschaftlichen Bereich. Welche Bedeutung ihr in dieser Hinsicht zukommt, erhellt am besten daraus, daß die Vereinigten Staaten sich damit Erdöl, Erdgas und andere Rohstoffe eines Teils des Meeresbodens sicherten, der größer ist als das Gebiet der ursprünglichen 13 Kolonien und des Louisiana-Kaufs zusammen ${ }^{39}$. Vermutlich war es jedoch vor allem die außenwirtschaftliche Tragweite der Proklamation im Verhältnis zu den Staaten Lateinamerikas, die Art und Umfang der von einigen dieser Staaten in der Folgezeit ergriffenen Maßnahmen bestimmte. Aus der Sicht dieser sämtlich als Entwicklungsländer zu charakterisierenden Staaten vergrößerte sich nämlich

35 So die in den Anm. 2, 6 und 9 zitierten Vorschriften Argentiniens, El Salvadors und Perus.

36 Vgl. Art. 3; Fundstelle: Anm. 10.

37 Vgl. Art. 3; Fundstelle: Anm. 3 .

38 Englischer Text: ST/LEG/SER. B/1, S. 38.

39 Vgl. Hersh, Seymour M.: An Arms Race on the Sea Bed?, in: War/Peace Report, Bd. 8 (1968), S. 8-9, $21-22(8)$. 
infolge des hierdurch eingetretenen beträchtlichen Zuwachses an Bodenschätzen auf seiten der Vereinigten Staaten ihr wirtschaftlicher Rückstand gegenüber dem reichen Nachbarn im Norden beträchtlich, da sie selbst infolge ihres Entwicklungsstandes den ihren Küsten vorgelagerten Festlandsockel nicht ausbeuten konnten und deshalb fast so arm blieben wie vorher. Besonders die Staaten an der Westküste, die wegen des steilen Abfalls ihrer Küsten in den Pazifik ohnehin nur über einen äußerst schmalen Festlandsockel verfügen, konnten sich deswegen nicht nur mit einem gewissen Recht benachteiligt fühlen. Sie mußten auch bestrebt sein, in geeigneter Weise gleichzuziehen, wenn die Proklamation Präsident Trumans nicht einen Rückschlag bei ihren Bemühungen zur Beseitigung der wirtschaftlichen Abhängigkeit von den Vereinigten Staaten bedeuten sollte.

Die Richtung für derartige Bestrebungen hatte der amerikanische Präsident in einer zweiten - heute noch gültigen - Proklamation gleichen Datums ${ }^{40}$, in der die Errichtung von Fischereischutzzonen auf dem an das Küstenmeer der Vereinigten Staaten angrenzenden Hohen Meer angekündigt wurde, selbst gewiesen. Fischfang und andere Methoden der Ausbeutung der lebenden Naturschätze des Meeres waren nämlich die Formen der Nutzung des Meeres, die dem damaligen Entwicklungsstand dieser Staaten am ehesten entsprachen. Der gewünschte wirtschaftliche Ausgleich für die fehlende Möglichkeit der Ausbeutung des Festlandsockels, bzw. für dessen Fehlen überhaupt konnte jedoch nur erreicht werden, wenn man sich nicht auf die Errichtung von Fischereischutzzonen beschränkte, sondern dem Beispiel der Festlandsockel-Proklamation der Vereinigten Staaten folgend Monopolrechte außerhalb des Küstenmeeres in Anspruch nahm.

Einige Staaten griffen daher die bereits aus der Zeit vor dem Ersten Weltkrieg stammende, unter anderem auch von argentinischen Juristen vertretene Theorie von der Gebietshoheit des Küstenstaates über das epikontinentale Meer41 auf und beanspruchten Souveränität, bzw. ausschließliche Fischereirechte in dem über dem Festlandsockel liegenden Teil des Hohen Meeres ${ }^{42}$. Für Staaten wie Chile und Peru erbrachte diese Theorie aber keinen Zuwachs an wirtschaftlich verwertbaren Rechten, da vor ihren Küsten ein Festlandsockel praktisch fehlt. Sie erhoben daher zunächst auf ausschließliche Fischereirechte beschränkte Hoheitsansprüche in einer an die Küste angrenzenden Zone von $200 \mathrm{sm} \mathrm{Breite}{ }^{43}$. Von dort bis zur Geltendmachung von Ansprüchen auf ein Küstenmeer, dessen Breite weit über die traditionelle 3-sm-Grenze hinausgeht, war es dann nur noch ein kleiner Schritt. Nachdem bereits am 21. Januar 1948 Chile sein Küstenmeer auf $50 \mathrm{~km}$ Breite ausgedehnt hatte ${ }^{44}$, nahm El Salvador am 7. September 1950 in seine Verfassung einen Artikel auf, der die Breite des Küstenmeeres zum ersten Mal sogar auf 200 sm festsetzte ${ }^{45}$.

Damit war jedoch für alle Staaten sichtbar der Bogen überspannt worden. Im Gegensatz zur Festlandsockel-Proklamation, die - zum Teil wegen gleichgerichteter Wirtschaftsinteressen - noch widerspruchslos hingenommen worden war, obwohl sie die Bodenschätze eines beachtlichen Teils des Hohen Meeres der Aus-

40 Proklamation Nr. 2668 vom 28. 9. 1945; englischer Text: ST/LEG/SER. B/1, S. 112.

$41 \mathrm{Vgl}$. Kunz, Josef. L.: Festlandsockel, in: Wörterbuch I, S. 516-520 (516).

42 Vgl. u. a. die Deklaration des mexikanischen Präsidenten vom 29. 10. 1945 (englischer Text: ST/LEG/SER. $\mathrm{B} / 1$, S. 13); das argentinische Dekret Nr. 14.708 vom 11. 10. 1946 (englischer Text: ST/LEG/SER. B/1, S. 4) und das panamesische Dekret Nr. 449 vom 17. 12. 1946 (englischer Text: ST/LEG/SER. B/1, S. 16).

43 Vgl. u. a. die Deklaration des chilenischen Präsidenten vom 23. 6. 1947 (englischer Text: ST/LEG/SER. B/1, S. 6) und das peruanische Dekret Nr. 781 vom 1. 8. 1947 (englischer Text: ST/LEG/SER. B/1, S. 16).

44 Vgl. Gesetz Nr. 8.944; englischer Text: ST/LEG/SER. B/1, S. 61.

45 Vgl. Art. 7; englischer Text: Peaslee, Amos J.: Constitutions of Nations, 2. Aufl., Bd. 1, Den Haag 1956, S. 818 . 
beutung durch die übrigen Staaten entzogen hatte ${ }^{46}$, führten daher die Maßnahmen der lateinamerikanischen Staaten zu Protesten und zu zahlreichen Zwischenfällen ${ }^{47}$. Vor allem die Vereinigten Staaten, die sich selbst bei der Geltendmachung ihres Anspruches auf die Naturschätze des ihren Küsten vorgelagerten Festlandsockels in eklatanter Weise über das Prinzip der Freiheit der Meere hinweggesetzt hatten, protestierten namentlich gegen die Beeinträchtigung ihrer Fischereiinteressen ${ }^{48}$. Sie schafften damit die Grundlage für die seit Anfang der fünfziger Jahre zu beobachtende, ständig fortschreitende Solidarisierung der mittel- und südamerikanischen Staaten ${ }^{49}$, welche auch zum Scheitern aller Versuche zur Festlegung der äußersten Küstenmeerbreite auf den Genfer Seerechtskonferenzen wesentlich mit beigetragen haben dürfte. Wie die Vereinigten Staaten, so zeigten aber auch viele andere Staaten, die keine Bedenken gegen die Einschränkung der Freiheit des Hohen Meeres durch Inanspruchnahme von Monopolrechten an dem ihren Küsten vorgelagerten Festlandsockel gehabt hatten, in der Folgezeit nicht den politischen Weitblick, der erforderlich gewesen wäre, um den Küstenstaaten, die von der Nutzung des Festlandsockels nicht ebenfalls profitieren konnten, rechtzeitig einen Ausgleich in Gestalt begrenzter ausschließlicher Fischereirechte in den an ihr Küstenmeer angrenzenden Teilen des Hohen Meeres zuzugestehen und dadurch die Ansprüche der lateinamerikanischen Staaten auf ein erträgliches Maß zurückzuschrauben.

Das hat dazu geführt, daß seit Mitte der sechziger Jahre die Zahl der Staaten, die volle oder inhaltlich beschränkte Hoheitsrechte in der 200-sm-Zone beanspruchen, weiter gestiegen ist ${ }^{50}$. Auch Staaten an der südamerikanischen Ostküste machen jetzt derart weitgehende Rechte geltend, obwohl die geographischen Gegebenheiten hier grundverschieden von denen der Westküste sind ${ }^{51}$. Gegenüber den fünfziger Jahren hat sich die Situation darüber hinaus dadurch verschlechtert, daß die Entwicklung eine gewisse Eigengesetzlichkeit entfaltet hat und jetzt auch aus anderen Gründen vorangetrieben wird. Die jüngste Ausdehnung des brasilianischen Küstenmeers auf $200 \mathrm{sm}^{52}$ etwa unterstützt zwar politisch die entsprechenden Ansprüche anderer lateinamerikanischer Staaten, verfolgt aber gleichzeitig auch handfeste nationale Wirtschaftsinteressen ${ }^{53}$. Besonders verhängnisvoll erscheinen die Impulse, die von der noch in diesem Jahrzehnt beginnenden Ausbeutung der Rohstoffe des Bodens und Untergrundes der Tiefsee ausgehen, da dieses Phänomen weit über die 200-sm-Zone hinausweist ${ }^{54}$.

Insofern dokumentiert die Deklaration von Montevideo nicht nur den Grad der derzeitigen Solidarisierung unter den Staaten Lateinamerikas, sondern sie deutet darüber hinaus an, daß die 200-sm-Zone durchaus nicht der Endpunkt der Entwick-

46 Die Maßnahme stand deshalb zunächst in Widerspruch zum damaligen Seevölkerrecht und wurde erst nachträglich durch die Herausbildung entsprechender neuer völkerrechtlicher Regeln legalisiert; vgl. Kunz, S. 518 .

47 Vgl. die Zusammenstellung bei Rojahn, Ondolf: Die Berechtigung der Ansprüche auf ein Küstenmeer von mehr als $12 \mathrm{sm}$ Breite, in: Kehden-Henkmann: Die Inanspruchnahme von Meereszonen durch Küstenstaaten, 1. Aufl., Hamburg 1967, S. 277-306 (283 f.).

48 Vgl. die Noten an Argentinien, Chile und Peru vom 2. 7. 1948; englischer Text: ST/LEG/SER. B/1, S. 5,7 und 17 .

49 Den Anfang bildete die Gemeinsame Seezonen-Erklärung der Staaten Chile, Ecuador und Peru vom 18. 8. 1952; englischer Text: ST/LEG/SER. B/6, S. 723.

$50 \mathrm{Vgl}$. die Úbersicht auf S. 521.

51 Der Umstand, daß zum Beispiel Peru Hoheitsrechte in einer Zone von ausgerechnet $200 \mathrm{sm}$ Breite beansprucht, erklärt sich daraus, daß nur so der fischreiche Humboldt-Strom, der in größerer Entfernung von der Küste verläuft, erfaßt wird. Der Ostküste ist dagegen ein breiter Festlandsockel vorgelagert, woraus sich völlig andere ökologische Bedingungen ergeben.

52 Vgl. Anm. 3.

53 Vgl. The New York Times vom 26. 3. 1970.

54 Das dürfte auch der Grund sein, weshalb die Signatarstaaten der Deklaration von Montevideo keine große Neigung zur Neubestimmung, d. h. Begrenzung der Festlandsockelrechte zeigen; vgl. S. 526. 
lung zu sein braucht und gar eine Rückkehr zu schmaleren Hoheitszonen nur dann denkbar erscheint, wenn man sich bald hierüber einigen kann. Beide Gesichtspunkte zusammen führen zu einer weiteren Bedeutung der Deklaration hin, die die weitere Entwicklung wahrscheinlich nachhaltig beeinflussen wird: Die Signatarstaaten haben sich durch die Deklaration verbal in einer Weise exponiert, daß es ihnen schon aus Prestigegründen nicht mehr möglich ist, ohne Zugeständnisse der übrigen Staaten ${ }^{55}$ die in der Deklaration aufgebauten Positionen wenigstens in der Sache zum Teil zu verlassen. Wie unter II. ausgeführt wurde, läßt die Deklaration zwar ein diesbezügliches Nachgeben der Signatarstaaten zu, insbesondere hinsichtlich der Maximalbreite des Küstenmeeres. Es wäre jedoch illusionär zu glauben, daß man um die Gewährung neuer Monopolrechte in dem an das Küstenmeer angrenzenden Teil des Hohen Meeres herumkommen wird. Wenn der sich danach anbietende Kompromiß der Beschränkung der Breite des Küstenmeers auf $12 \mathrm{sm}$ um den Preis ausschließlicher Fischerei- und Meeresbodennutzungsrechte in dem an das Küstenmeer angrenzenden Teil des Hohen Meeres aber nicht zustandekommt, werden die Signatarstaaten allerdings durch die Deklaration geradezu gezwungen, noch weitergehende Ansprüche zu erheben als bisher. Der Umstand, daß eine solche Entwicklung auch für sie nicht ohne Risiko wäre, dürfte ihre in der Deklaration versteckt zum Ausdruck gebrachte Kompromißbereitschaft jedoch weiter erhöhen. Die Deklaration vergrößert also paradoxerweise im Ergebnis sowohl die Erfolgsaussichten einer dritten Seerechtskonferenz als auch die negativen Aspekte ihres Scheiterns.

Abschließend läßt sich schon heute voraussagen, daß die Konferenz nur dann "erfolgreich“ verlaufen wird, wenn man sich auf weitere Einschränkungen der Freiheit der Meere einigen kann. Das ist vor allem deshalb bedauerlich, weil die Ausdehnung küstenstaatlicher Rechte nicht der geeignete Weg zur Bewältigung der Zukunftsprobleme der Meeresnutzungen ist. Fairerweise muß man jedoch anerkennen, daß nach Durchsetzung der Festlandsockelrechte ein Ausgleich zwischen den Küstenstaaten, die hierdurch wirtschaftlich begünstigt worden sind, und den übrigen fällig ist. Hierfür ist bedeutsam, daß eine wie auch immer geartete Tiefenlinie die Staaten im Hinblick auf die Abgrenzung der Monopolrechte zur Ausbeutung der Bodenschätze des Hohen Meeres nur formal gleichstellt. $\mathrm{Da}$ im übrigen die Staatenpraxis bei der Vergabe von diesbezüglichen Abbaukonzessionen bereits über den Festlandsockel im naturwissenschaftlichen Sinne hinausgreift, erscheint deshalb der Zeitpunkt gekommen, die Ausbeutung der Vorkommen des Bodens und Untergrundes des Hohen Meeres in der Weise neu zu regeln, daß Meeresbodenzonen bestimmter, für alle Küstenstaaten gleicher Breite diesen zur ausschließlichen Nutzung zugewiesen werden. Da selbst eine derartige Regelung bestimmte Staaten infolge ihrer geographischen Gegebenheiten und ihres Entwicklungsstandes immer noch wirtschaftlich benachteiligen würde, muß der Ausgleich aber auch die Gewährung ausschließlicher Nutzungsrechte an den lebenden Naturschätzen der an das Küstenmeer der betreffenden Staaten angrenzenden Teile des Hohen Meeres einschließen. Die dritte Seerechtskonferenz wird also nur dann zu einer Festlegung der Küstenmeerbreite führen, wenn es gelingt, eine Formel für die Schaffung von Monopol- oder Vorzugsrechten der Küstenstaaten an den Naturschät-

55 Diese erscheinen allerdings nicht mehr ausgeschlossen. Insbesondere die Vereinigten Staaten nehmen jetzt eine flexiblere Haltung ein; vgl. Anm. 16. 
zen der an ihr Küstenmeer angrenzenden Teile des Hohen Meeres sowie seines Bodens und Untergrundes zu finden, die alle Küstenstaaten wirtschaftlich in gleichem Maße begünstigt. Die eigentliche Schwierigkeit liegt dabei darin, darüber hinaus den berechtigten Interessen der Staatengemeinschaft an der Aufrechterhaltung einer nicht lediglich in der Freiheit der Schiffahrt und des Flugverkehrs bestehenden Freiheit der Meere Rechnung zu tragen.

\section{Deklaration von Montevideo über das Seerecht *}

Die auf der Zusammenkunft von Montevideo über das Seerecht vertretenen Staaten,

In Anerkennung des Bestehens eines geographischen, wirtschaftlichen und sozialen Zusammenhangs zwischen der See, dem Land und seinen Bewohnern, aus dem sich ein rechtmäßiger Vorrang der Küstenbewohner zur Nutzung der Naturschätze ergibt, die ihre maritime Umwelt ihnen bietet;

In Anerkennung zugleich der Tatsache, daß die Regeln über die Grenzen nationaler Souveränität und Hoheit auf dem Meer, dem Meeresboden und -untergrund sowie über die Modalitäten der Ausbeutung der Meeresschätze die geographischen Gegebenheiten der Küstenstaaten wie auch die besonderen Notwendigkeiten und die wirtschaftlichen und sozialen Verpflichtungen der Entwicklungsländer berücksichtigen müssen;

In der Erwägung: daß der wissenschaftliche und technologische Fortschritt bei der Ausbeutung der Naturschätze des Meeres die Gefahr einer Erschöpfung der biologischen Hilfsquellen durch unvernünftige und mißbräuchliche Ausbeutungsmethoden oder durch Störung der ökologischen Bedingungen geschaffen hat, was den Küstenstaaten das Recht gibt, die erforderlichen Maßnahmen zum Schutze besagter Schätze in ausgedehnteren Hoheitszonen als den herkömmlichen zu ergreifen und in den genannten Zonen die Fischerei und die maritime Jagd durch Schiffe nationaler oder fremder Flagge mittels Unterwerfung unter die innerstaatlichen Gesetze oder etwaige Abkommen mit anderen Staaten zu regeln;

$\mathrm{Da}$ in - vor allem interamerikanischen - Deklarationen, Resolutionen und Verträgen ebenso wie in multilateralen Deklarationen und Abkommen zwischen Staaten Lateinamerikas rechtliche Prinzipien verankert sind, die das Recht der Küstenstaaten belegen, ihre Souveränität und Hoheit in dem Maße auszudehnen, wie es zur Erhaltung, Entfaltung und Ausbeutung der Naturschätze der an ihre Küsten angrenzenden Meereszone, ihres Bodens und Untergrundes erforderlich ist;

*) Deutsche Ubersetzung des Autors. 
$\mathrm{Da}$ in Übereinstimmung mit den genannten rechtlichen Prinzipien die unterzeichneten Staaten aufgrund ihrer besonderen Bedingungen ihre Souveränität oder ihre ausschließlichen Hoheitsrechte über die an ihre Küsten angrenzende Meereszone, deren Boden und Untergrund bis zu einer Entfernung von 200 Seemeilen, von der Basislinie des Küstenmeeres aus gerechnet, ausgedehnt haben;

$\mathrm{Daß}$ die Anwendung von Maßnahmen zur Erhaltung der Schätze des Meeres, des Meeresbodens und -untergrundes durch die Küstenstaaten in den an ihre Küsten angrenzenden, ihrer Hoheit unterliegenden Meereszonen letztlich der ganzen Menschheit, die in den Ozeanen eine Hauptquelle von Hilfsmitteln für ihre Existenz und Entwicklung besitzt, zum Nutzen gereicht;

$\mathrm{Daß}$ das souveräne Recht der Staaten auf ihre Naturschätze durch zahlreiche Resolutionen der Vollversammlung und anderer Organe der Vereinten Nationen anerkannt und bekräftigt worden ist;

$\mathrm{Da} ß$ es angebracht ist, in einer gemeinsamen Erklärung die Grundsätze zu bestimmen, die sich aus den neuen Tendenzen zur Ausbildung eines Völkerrechts ergeben, welches deutlich in fortschrittlicher Entwicklung begriffen ist und eine ständig zunehmende Unterstützung durch die internationale Gemeinschaft erfährt;

Erklären zu grundlegenden Prinzipien des Seerechts:

1. Das Recht der Küstenstaaten, über die Naturschätze des an ihre Küsten angrenzenden Meeres und des Bodens und Untergrundes desselben Meeres zu verfügen, um die maximale Entwicklung ihrer Volkswirtschaften zu fördern und den Lebensstandard ihrer Völker zu heben;

2. Das Recht, die seewärtigen Grenzen ihrer Souveränität und Hoheit übereinstimmend mit ihren geographischen und geologischen Eigenheiten und mit den Gesichtspunkten, von denen das Vorhandensein der Meeresschätze und die Notwendigkeit ihrer vernünftigen Ausnutzung abhängen, festzusetzen;

3. Das Recht, die lebenden Schätze des an ihr Staatsgebiet angrenzenden Meeres $\mathrm{zu}$ erforschen und $\mathrm{zu}$ erhalten sowie den Fischfang und die maritime Jagd gesetzlich zu regeln;

4. Das Recht, die Naturschätze ihrer jeweiligen Festlandsockel zu erforschen, zu erhalten und auszubeuten, soweit die Tiefe des darüber befindlichen Wassers die Ausbeutung besagter Schätze gestattet;

5. Das Recht, die Naturschätze des Bodens und Untergrundes der Meerestiefen bis zur Hoheitsgrenze des Küstenstaates zu erforschen, zu erhalten und auszubeuten;

6. Das Recht, zu den vorbenannten Zwecken Bestimmungen zu erlassen, die ohne Beeinträchtigung der Freiheit der Schiffahrt und des Flugverkehrs für Schiffe und Flugzeuge beliebiger Nationalität in den ihrer Souveränität und Hoheit unterliegenden Meereszonen gelten sollen. 
Ermutigt durch die Ergebnisse dieser Zusammenkunft, erklären die unterzeichnenden Staaten außerdem ihre Absicht, ihre zukünftige Tätigkeit zu koordinieren, um die wirksame Verteidigung der in dieser Deklaration aufgeführten Prinzipien sicherzustellen.

Diese Deklaration wird bezeichnet werden als „Deklaration von Montevideo über das Seerecht". 